\title{
Expression of MLL-AF4 or AF4-MLL fusions does not impact the efficiency of DNA damage repair
}

\author{
Julio Castaño', Ana B. Herrero², Aldeheid Bursen ${ }^{3}$, Federico González ${ }^{4}$, Rolf \\ Marschalek $^{3}$, Norma C. Gutiérrez ${ }^{2}$ and Pablo Menendez ${ }^{1,5}$ \\ 1 Josep Carreras Leukemia Research Institute, Department of Biomedicine, School of Medicine, University of Barcelona, \\ Barcelona, Spain \\ ${ }^{2}$ Hematology Department, University Hospital of Salamanca, IBSAL, IBMCC (USAL-CSIC), Salamanca, Spain \\ ${ }^{3}$ Institute Pharmaceutical Biology, Goethe-University, Frankfurt/Main, Germany \\ ${ }^{4}$ IBEC - Institute for Bioengineering of Catalonia, Barcelona, Spain \\ ${ }^{5}$ Institució Catalana de Recerca i Estudis Avançats (ICREA), Barcelona, Spain \\ Correspondence to: Pablo Menendez, email: pmenendez@carrerasresearch.org \\ Keywords: MLL.AF4, AF4.MLL, †(4;1 1), DSB, infant leukemia \\ Received: April 11,2016 Accepted: April 13,2016 Published: April 22, 2016
}

\section{ABSTRACT}

The most frequent rearrangement of the human MLL gene fuses MLL to AF4 resulting in high-risk infant B-cell acute lymphoblastic leukemia (B-ALL). MLL fusions are also hallmark oncogenic events in secondary acute myeloid leukemia. They are a direct consequence of mis-repaired DNA double strand breaks (DNA-DSBs) due to defects in the DNA damage response associated with exposure to topoisomeraseII poisons such as etoposide. It has been suggested that MLL fusions render cells susceptible to additional chromosomal damage upon exposure to etoposide. Conversely, the genome-wide mutational landscape in MLL-rearranged infant B-ALL has been reported silent. Thus, whether MLL fusions compromise the recognition and/ or repair of DNA damage remains unanswered. Here, the fusion proteins MLL-AF4 (MA4) and AF4-MLL (A4M) were CRISPR/Cas9-genome edited in the AAVS1 locus of HEK293 cells as a model to study MLL fusion-mediated DNA-DSB formation/repair. Repair kinetics of etoposide- and ionizing radiation-induced DSBs was identical in WT, MA4- and A4M-expressing cells, as revealed by flow cytometry, by immunoblot for YH2AX and by comet assay. Accordingly, no differences were observed between WT, MA4- and A4M-expressing cells in the presence of master proteins involved in non-homologous end-joining (NHEJ; i.e.KU86, KU70), alternative-NHEJ (Alt-NHEJ; i.e.LigIIIa, WRN and PARP1), and homologous recombination (HR, i.e.RAD51). Moreover, functional assays revealed identical NHEJ and HR efficiency irrespective of the genotype. Treatment with etoposide consistently induced cell cycle arrest in S/ G2/M independent of MA4/A4M expression, revealing a proper activation of the DNA damage checkpoints. Collectively, expression of MA4 or A4M does neither influence DNA signaling nor DNA-DSB repair.

\section{INTRODUCTION}

The mixed-lineage leukemia (MLL) gene fuses to generate chimeric genes with 80 partners in human leukemia [1]. Infant pro-B acute lymphoblastic leukemia (B-ALL) harboring the fusion MLL-AF4 (MA4) represents a rare leukemia, associated with very brief latency and dismal prognosis, raising the question of how this disease evolves so quickly [2]. Epidemiological and genetic studies support the contention that the in utero origin of MA4 in infant B-ALL may be the result of transplacental exposures during pregnancy to 
quinone-based chemicals or dietary flavonoids [3-5]. This parallels the origin of therapy-related "secondary" leukemias harboring $M L L$ - rearrangements $[4,6,7]$ and is supported by the finding that $M L L$ gene rearrangements can be induced in vitro and in vivo in hematopoietic stem/ progenitor cells (HSPCs) at different ontogeny stages by etoposide, a topoisomerase-II inhibitor commonly used in chemotherapy regimens [8-11].

Exposure to environmental agents may represent a potential etiological driver in $M L L$-rearranged leukemia. $M L L$ - rearrangements are the consequence of misrepaired DNA double strand breaks (DNA-DSBs) [12, 13]. Alternatively, they might be due to defects in the DNA damage response (DDR) after chronic exposure to topoisomerase-II poisons (etoposide, bioflavonoids, and pesticides) or even irradiation in early HSPCs [3-5, 7, 14]. Additionally, overexpressed MLL fusions have been shown to render cells more susceptible to additional chromosomal damage upon exposure to etoposide, suggesting that expression of MLL fusion proteins not only transform cells but also compromise the recognition and/or repair of DNA damage [15]. Contrasting this view are the recent findings deciphering a silent mutational genome-wide landscape in $M L L$-rearranged infant B-ALL [16-19]. Therefore, the genomic stability observed in $M L L$-r infant leukemia poses the question of whether or not the recognition and repair of DNA damage is influenced by MLL fusions. Here, we have attempted to address this question by exploiting a syngeneic human cell system in which a single copy of the fusion protein MA4 or A4M was CRISPR/Cas9-genome edited in the AAVS1 locus of HEK293 cells as a model to study MLL fusionmediated DNA-DSB formation and repair.

\section{RESULTS}

\section{CRISPR/Cas9-mediated insertion of MA4 and A4M into AAVS1 safe harbor}

To address whether the recognition and repair of DNA damage is regulated by MA4 and A4M expression, a single copy of each fusion was CRISPR/Cas9-genome edited in the AAVS1 locus of HEK293 human cells. Two donor vectors harboring dTo-MA4 or A4M-GFP cassettes under the transcriptional control of CAG promoter, and flanked by AAVS1 homology arms were generated (Figure 1A, 1F). These vectors contain a promoterless puromycin (Figure 1A) or neomycin (Figure 1F) cDNA preceded by a splice acceptor (SA) site and a translational self-cleaving 2A sequence. Successful Cas9-targeted insertion into AAVS1 locus confers transcription of both puromycin and neomycin cassettes under the control of the ubiquitously expressed $P P P 1 R 12 C$ promoter, thus allowing the antibiotic selection of the targeted cells. A
guide-RNA directed to AAVS1 locus was used to enhance the gene targeting efficiency. After antibiotic selection, many antibiotic-resistant clones were isolated and MA4- and A4M-targeted clones were $\mathrm{dTo}+$ and GFP+, respectively (Figure 1B, 1G). PCR analysis using primers specific for $5^{\prime}$ and $3^{\prime}$ integration junctions of the AAVS1 locus showed proper genomic integration for MA4 and A4M, respectively (Figure 1C, 1H). RT-PCR demonstrated specific RNA expression of MA4 and A4M transcripts in the targeted cells (Figure 1D, 1I). Importantly, southern blot analysis further confirmed a single copy correct integration of the donor vectors (MA4 and A4M cassettes, Figure 1E, 1J). Importantly, the expression level of MA4 in MA4-edited HEK293 cells was comparable with that one of four independent primary $\mathrm{t}(4 ; 11)+$ pediatric B-ALL (Figure 1K). Furthermore, the MA4 target genes HOXA9 and PROM1 were similarly upregulated upon either CRISPR/Cas9- or lentiviral-mediated integration/ expression (Figure 1K). Together, CRISPR/Cas9-genome edited isogenic cells were developed allowing us to address whether MA4 and A4M facilitate/impair DNADSB formation/repair.

\section{Expression of either MA4 or A4M does not affect DNA-DSB formation nor repair}

The most deleterious form of DNA lesions are DSBs. Mis-repair of DSBs leads to either cell death or genome rearrangements [20]. To investigate whether MA4 or A4M impact DNA-DSB formation and/or repair, we first analyzed the effect of etoposide, a bona fide topoisomerase-II poison, on cell proliferation and clonogenic survival. MTT proliferation assays revealed identical $\mathrm{IC}_{50}(\sim 1 \mu \mathrm{M})$ for etoposide, irrespective of the investigated genotype (Figure 2A). Similarly, clonogenic survival measured 12-days after etoposide treatment revealed no differences among genotypes (Figure 2B). To analyze the kinetics of DSB formation and DNA repair, we monitored the phosphorylation of Ser139-H2AX $(\gamma \mathrm{H} 2 \mathrm{AX})$ [21] every $3 \mathrm{~h}$ after treatment with $1 \mu \mathrm{M}$ etoposide. $\gamma \mathrm{H} 2 \mathrm{AX}$ signal was quantified by flow cytometry in WT, MA4and A4M-expressing cells. $\gamma \mathrm{H} 2 \mathrm{AX}$ intensity (MFI) and the proportion of $\gamma \mathrm{H} 2 \mathrm{AX}+$ cells reached their maximum $3 \mathrm{~h}$ after etoposide treatment and started to fall over the next $12 \mathrm{~h}$, again with identical kinetics irrespective of the investigated genotype (Figure 2C). To confirm the flow cytometry data, $\gamma \mathrm{H} 2 \mathrm{AX}$ expression was analyzed by Western Blot. $\gamma \mathrm{H} 2 \mathrm{AX}$ peaked $3 \mathrm{~h}$ after etoposide treatment and then fall over the next $12 \mathrm{~h}$, with similar kinetics for $W T$, MA4- and A4M-expressing cells (Figure 2D).

Next, we analyzed the repair kinetics of IR-induced DSBs using the neutral comet assay. Similar to the kinetics of $\gamma \mathrm{H} 2 \mathrm{AX}$ loss, no differences were found in the kinetics of DSBs repair between MA4, A4M and $W T$ cell lines (Figure 3A, 3B). For all the genotypes most of the 
A

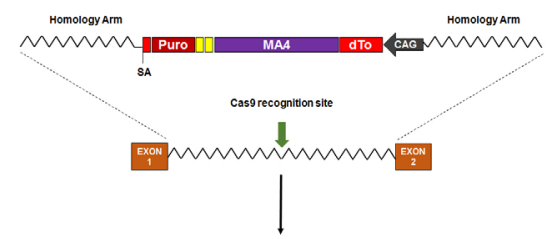

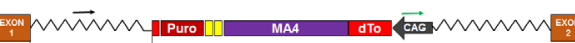

so

B

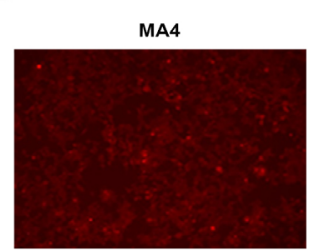

C

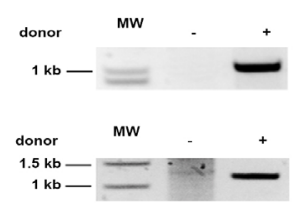

D

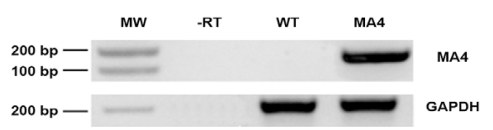

E

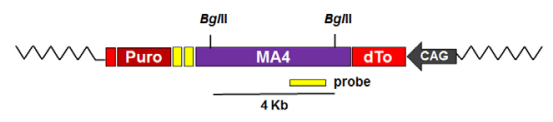

WT MA4

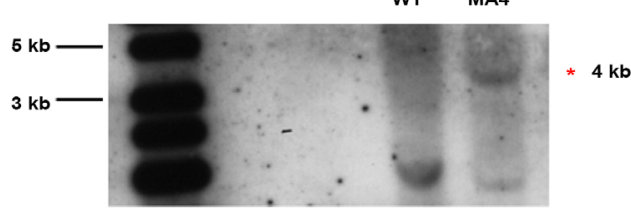

$\mathbf{F}$

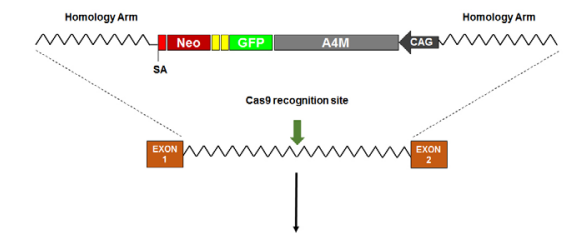

$\mathbf{G}$

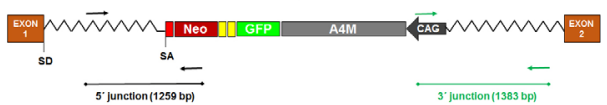

H

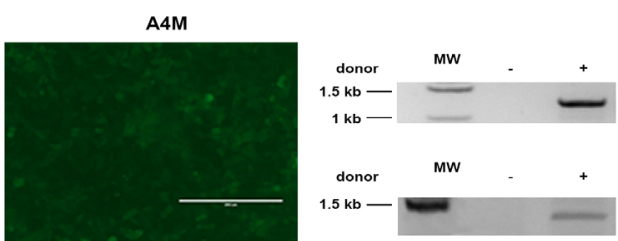

1

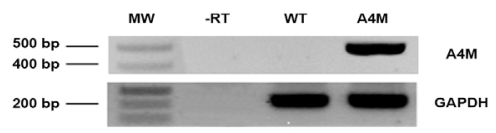

J

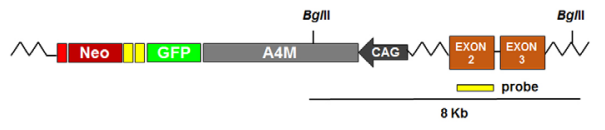

WT A4M

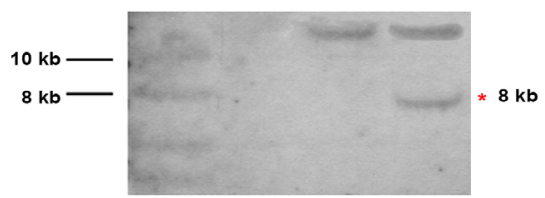

$\mathbf{L}$
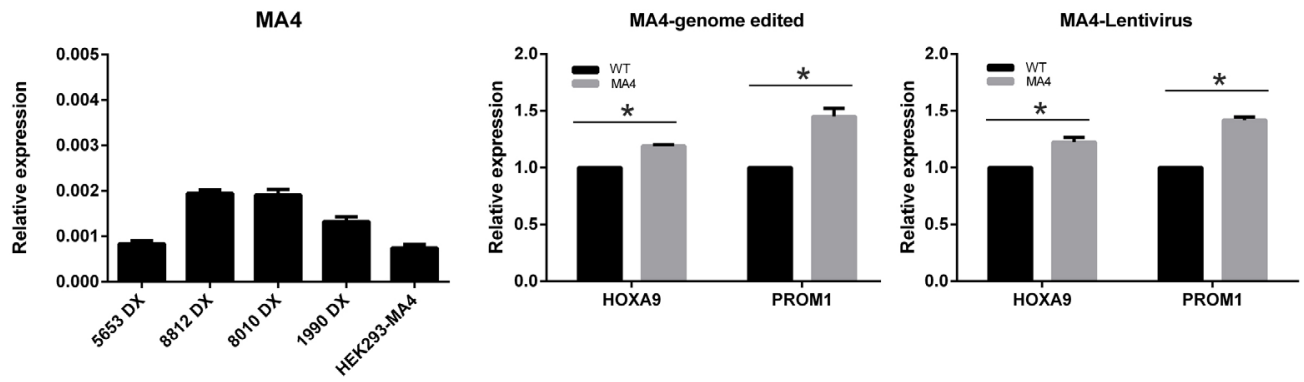

Figure 1: CRISPR/Cas9-mediated generation of syngeneic HEK293 cells expressing a unique copy of MA4 or A4M in the AAVS1 safe harbor. A., F. Schematic representation of the donor vector used for insertion of the dTo-MA4 A. or A4M-GFP F. cassette into the AAVS1 locus. dTo, dTomato fluorescent protein; SD, splice donor; SA, splice acceptor; CAG, CMV early enhancer/chicken $\beta$ actin promoter. Black (5' junction) and green ( 3 ' junction) arrows depict genomic location of primers used to confirm targeted integration. B.,G. Representative images of dTo-MA4- and A4M-GFP-expressing HEK293 cells after puromycin or G418 selection, respectively. C.,H. Targeted integration analysis of MA4 and A4M into the AAVS1 locus by PCR using primers specific for the 5' (top panels) and 3' (bottom panels) integration junctions. D.,I. RNA expression of MA4 D. and A4M I. in antibiotic-selected cells. E., J. Homologous recombination confirmed by southern blot analysis after BgIII digestion of genomic DNA from puromycin/G418-resistant clones using a MA4 probe $\mathbf{E}$. or an AAVS1 exon2 probe outside the targeting construct J.. A 4Kb band represents a targeted integration of MA4 in PPP1R12C. The 8kb band corresponds to the targeted integration of A4M in PPP1R12C. Untargeted allele gives a 12Kb band J.. L. qPCR of the MA4 targets HOXA9 and PROM1 is comparable between HEK293 cells ectopically expressing MA4 upon CRISPR/Cas9-mediated genome edition (left panel) or lentiviral transduction (right panel) ${ }^{*} p<0.05$, compared to $W T$. K. MA4 qPCR comparing B-ALL patients and HEK293MA4. 
DNA damage was repaired $6 \mathrm{~h}$ after IR, despite the high irradiation dose given (40 Gy) (Figure 3A, 3B). These data indicate that MA4- and A4M-expressing cells are capable of repairing most of both etoposide- and IR-induced DSBs similar to $W T$ syngeneic cells.

Cell cycle checkpoints are activated following induction of DSBs, providing time for the removal of the DNA damage [20]. In fact, etoposide induces G2/M checkpoints [22] which efficiently retain cells in G2 until they partially repair DSBs. Cell lines consistently showed a $\mathrm{G} 2 / \mathrm{M}$ arrest $9 \mathrm{~h}$ after etoposide treatment which was even more pronounced after $12 \mathrm{~h}$ (Figure 4). This G2/M arrest was independent of MA4 and A4M expression, revealing a proper activation of the DNA damage checkpoints.

\section{MA4 and A4M expression does not regulate either NHEJ or HR repair pathways}

To further confirm that MA4 and A4M do not affect DSB repair, we analyzed the steady-state levels of master proteins involved in the main pathways of DSB repair: NHEJ, HR and alternative NHEJ (Alt-NHEJ) [23]. Western blot analysis was performed in WT, MA4 and A4M cell lines (Figure 5). No differences were observed between $W T$, MA4- and A4M-expressing cells for the expression of proteins involved in NHEJ (KU86, KU70, DNA-PK ${ }_{\mathrm{cs}}$ and XRCC4, Figure 5A), Alt-NHEJ (LigIIIa, WRN and PARP1, Figure 5B), and HR (RAD51, Figure 5C).
A

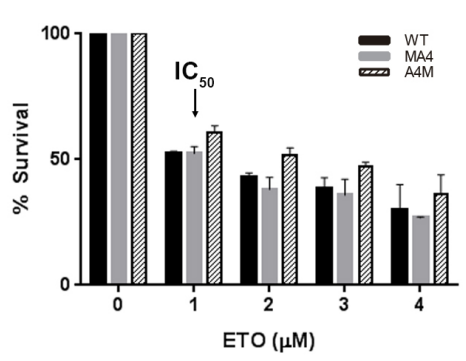

B

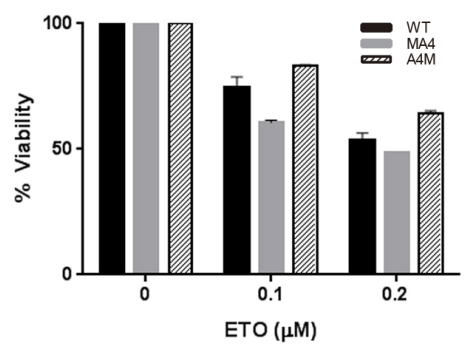

D

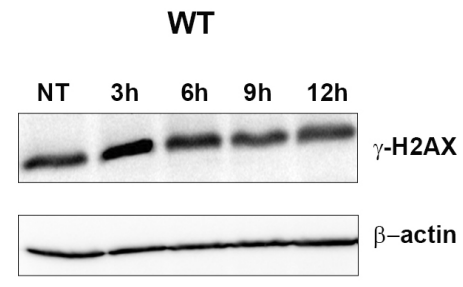

C
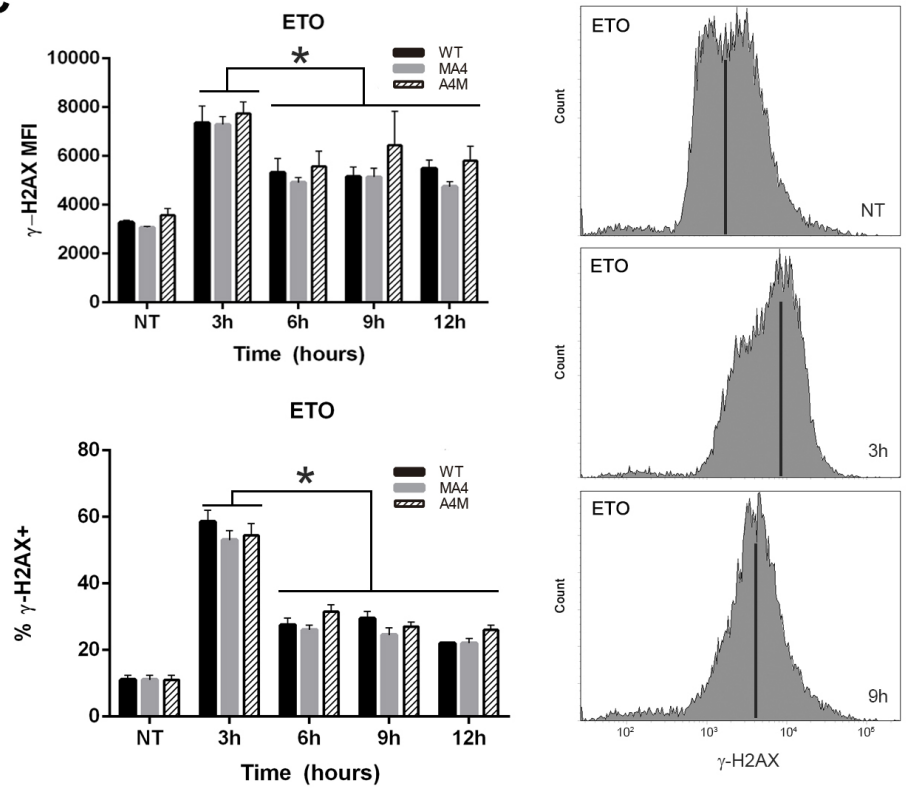

MA4

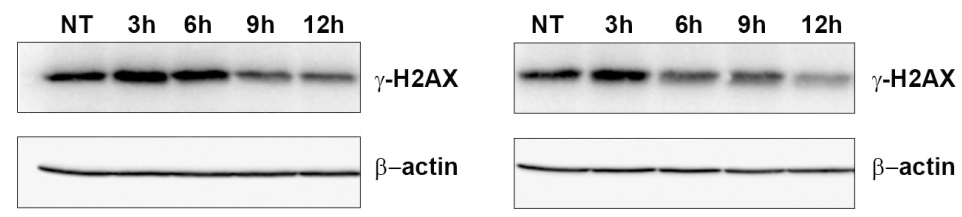

Figure 2: Kinetics of $\gamma \mathbf{H 2 A X}$ loss after etoposide treatment. A. Cell viability measured by MTT assay for increasing concentrations of etoposide $(n=3)$. The $\mathrm{IC}_{50}$ is $1 \mu \mathrm{M}$ irrespective of the genotype. B. Clonogenic survival measured 12-days after etoposide treatment revealing no differences between genotypes $(n=3)$. In A and B data is expressed as percentage of cell death relative to untreated control. C. Time course of $\gamma \mathrm{H} 2 \mathrm{AX}$ MFI (top left) and $\%$ of $\gamma \mathrm{H} 2 \mathrm{AX}+$ cells (bottom left) at the indicated time points after $1 \mu \mathrm{M}$ etoposide pulse $(n$ $=3$ ). Representative flow cytometry histograms of $\gamma \mathrm{H} 2 \mathrm{AX}$ staining in non-treated cells (NT) or cells $3 \mathrm{~h}$ and $9 \mathrm{~h}$ after $1 \mu \mathrm{M}$ etoposide pulse (right panel). D. Phospho- $\gamma \mathrm{H} 2 \mathrm{AX}$ western blot at different time points after etoposide treatment. $\beta$-actin was used as loading control. * $p<$ 0.05 , compared to NT. 
A

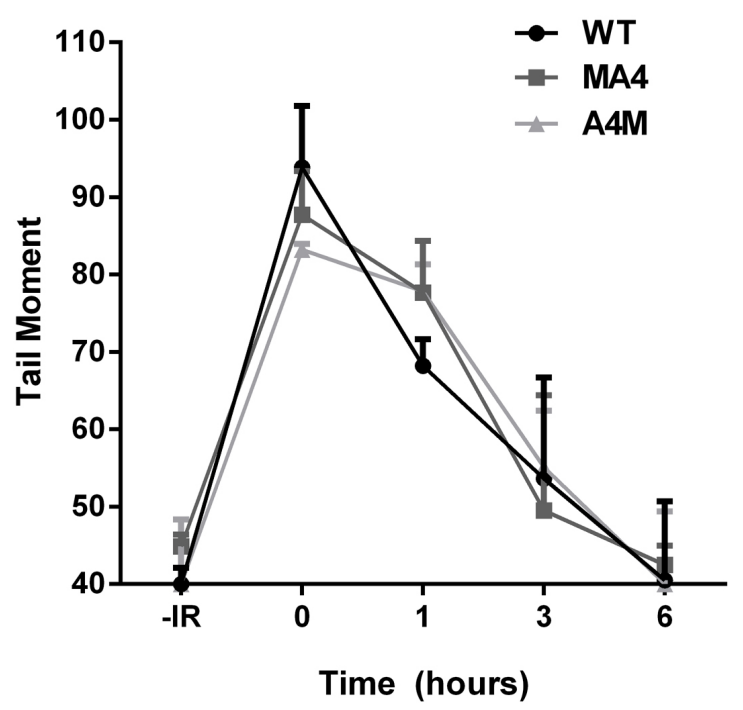

B

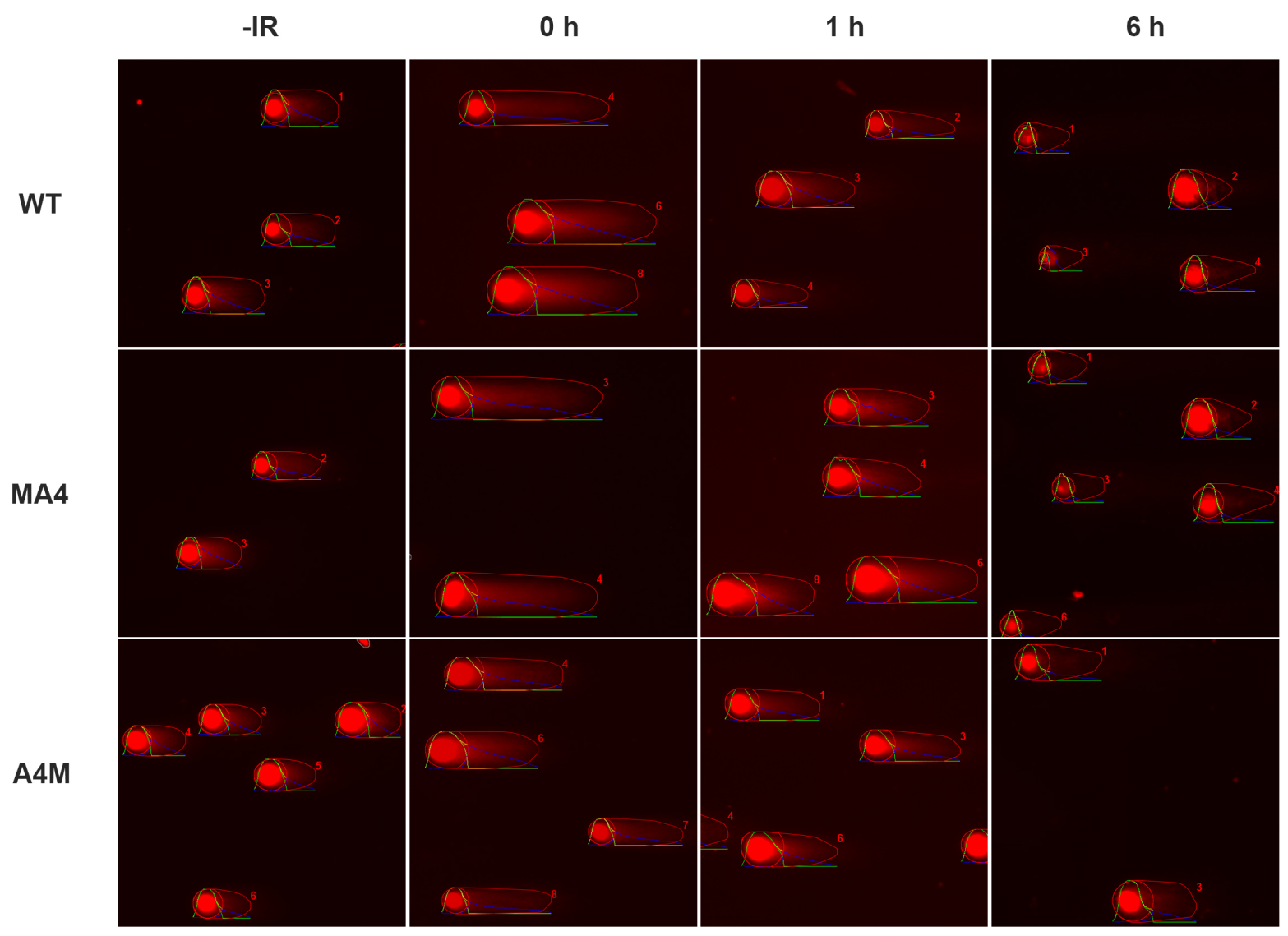

Figure 3: Analysis of DSB repair by neutral comet assay. A. $W T$, MA4 and A4M cells were irradiated with $40 \mathrm{~Gy}$ and the mean tail moment calculated using the OpenComet software. Data represent values of at least 75 comets in each of two independent experiments. B. Representative images of the comet assay. 
To further investigate the efficiency of NHEJ and $\mathrm{HR}$, functional assays were conducted by measuring the ability of the target cells to re-circularize HindIII- or SceI-digested pEGFP-Pem1-Ad2 NHEJ plasmid (Figure 6A) and SceI-digested HR reporter plasmid (Figure 6D). Successful re-ligation by the cell of enzyme-digested NHEJ or HR plasmids restores the expression of GFP. Thus, the percentage of GFP+ cells is a bona fide indicator of successful repair of DSBs by either NHEJ or HR. pDSRed2-N1 circular plasmid was always used to correct the transfection efficiency (Figure 6B, 6E). The proportion of GFP+ cells was almost identical in WT, MA4 and A4M cell lines, indicating identical NHEJ and HR efficiency irrespective of the investigated genotype (Figure 6C, 6F). Collectively, expression of either MA4 or A4M does not seem to influence either DNA signaling or DNA damage repair.

\section{DISCUSSION}

$M L L$-rearrangements are common in de novo infant acute leukemia and in therapy-related AML secondary to treatment with topoisomerase-II inhibitors (i.e. etoposide) [6]. Similar to IR-driven damage, topoisomerase-II poisons induce DSBs which are the most deleterious form of DNA lesions in living organisms. Mainly cells use the NHEJmediated DNA repair mechanism in G0 and G1 [24], while using $\mathrm{HR}$ in replicating cells or G2 where a proper template becomes available. Depending on the amount of
DSBs in a given cell, DSBs may compromise cell viability and will lead to cell death [20]. Assuming the cells remain usually in G0/G1, NHEJ-mediated DNA repair processes are more important. However, this type of repair frequently end in small deletion, duplications or inversions [12, 13]. Therefore, $M L L$-rearrangements can still be assumed as a consequence of mis-guided DNA repair [25]. In addition, exposure to topoisomerase-II poisons such as etoposide and bioflavonoids will increase the rate of DSBs, and thus, increase the probability of misguided DNA repair, resulting in chromosomal translocations [3-5, 7, 14]. Noteworthy, MLL-ENL fusions have been proposed to confer further susceptibility to chromosomal damage upon exposure to etoposide, suggesting that expression of MLL fusions not only transform cells but might also compromise the recognition and/or repair of DNA damage [15]. Similarly, MLL fusions have been found to alter cell cycle dynamics $[26,27]$ and to suppress DNA damage induced apoptosis mediated by TP53 [28]. Furthermore, specific transcriptional programs, including those involved in DDR, are frequently deregulated by various oncogenic transcription factors and chimeric genes. In contrast, recent findings revealed a silent mutational genome-wide landscape in $M L L$-rearranged infant B-ALL [16-19]. The genomic stability observed in $M L L-r$ infant leukemia poses then the question of whether or not MLL fusions render cells more vulnerable to further DNA damage and mutations through deregulation of DDR signaling. Here, we harnessed CRISPR/Cas9-based genome editing to
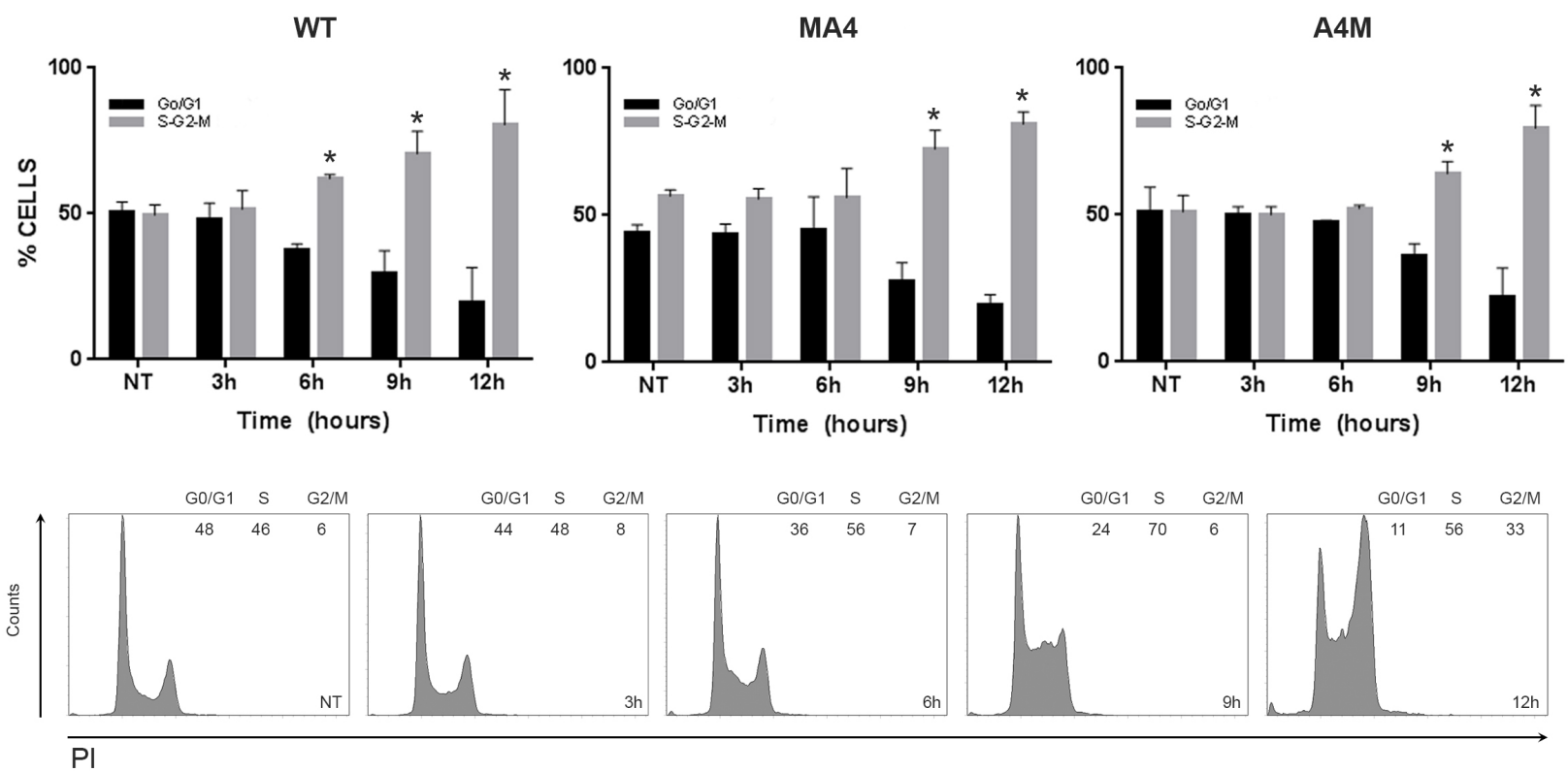

Figure 4: Cell cycle distribution assessed at the indicated time points after etoposide pulse. Top panels represent the \% of cells in G0/G1 vs S/G2/M cell cycle phases analyzed by FACS for the indicated genotypes $(n=3)$. Bottom panels are the corresponding flow cytometry histograms. Data represents mean $\pm \mathrm{SD}$ of three independent experiments $* p<0.05$. 
develop a system harboring either MA4 or A4M in the AAVS1 safe harbor of HEK293 cells. This model was useful to explore the influence of MLL fusion proteins on the amount of inducible DNA DSBs and the fidelity of the subsequent DNA repair process. The established cell culture model is unique because of the following: i) it is a syngeneic cellular model, ii) cells carry a single copy of each MLL fusion, and iii) the integration in a safe harbor ensures stable expression of both MA4 and A4M.

Repair kinetics of etoposide- and IR-induced DSBs was identical in WT, MA4- and A4M-expressing cells, as revealed by flow cytometry, by immunoblot for $\gamma \mathrm{H} 2 \mathrm{AX}$ and by the comet assay. In addition, functional assays revealed identical NHEJ and HR efficiency irrespective of the investigated genotype. These results indicate that although topoisomerase-II poisons may induce $M L L$ -rearrangements, these MLL chimeric proteins do not subsequently render cells more vulnerable to further DNA damage. In other words, MA4- or A4M-expressing cells that have survived exposure to genotoxic etoposide are equally likely to carry further DSBs than $W T$ cells. Molecular cytogenetic techniques were not used to analyze metaphases/interphases of cells exposed to etoposide. However, the absence of persistent DSBs and the similar NHEJ and HR repair efficiencies make specific chromosomal changes (structural and numerical

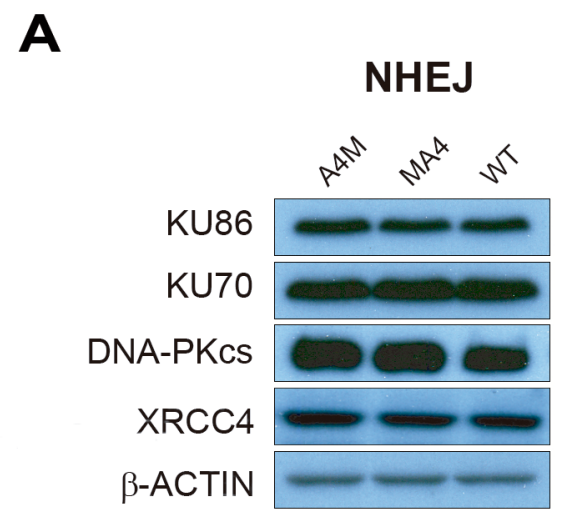

B
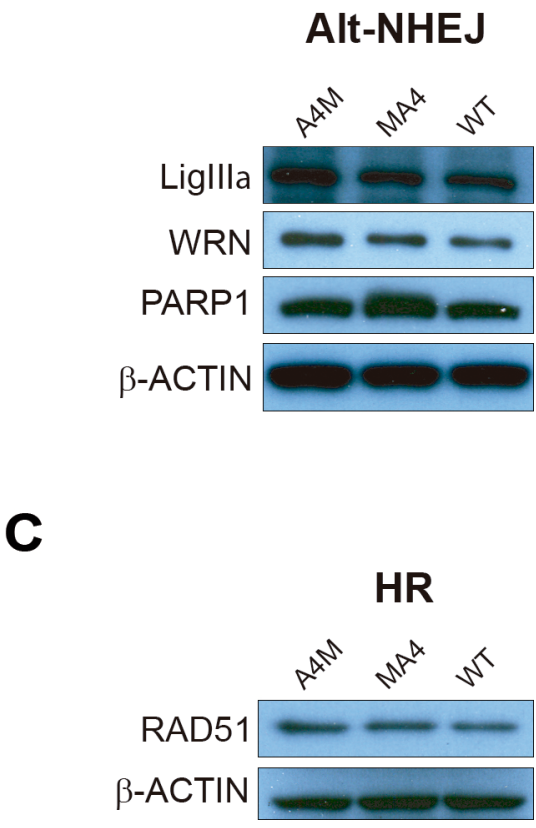

Figure 5: Western blot analysis of proteins involved in DSB repair. Proteins involved in the classical NHEJ pathway A., AltNHEJ proteins B. and the HR protein $\operatorname{Rad} 51$ C. are shown. 
alterations) or double minutes unlikely to occur since persistent DSBs must precede further DNA damage. One limitation of our study is the use of the HEK293 instead of hematopoietic cells. Of note, however, any hematopoietic immortalized cell line would carry important basal genomic instability similar to human kidney-derived 293 cells. The ideal scenario to address this question would rely on the generation of CRISPR/Cas9-genome edited primary CD34+ progenitors. However, reporter/ antibiotic selection of primary CD34+ progenitors carrying the functional $t(4 ; 11)$ is challenging [29-31], and using syngeneic cells is an asset to reduce (epi)-genetic variability among target cells.

Cell cycle checkpoints are activated following induction of DSBs, providing time for the removal of the DNA damage [20]. Etoposide induces G2/M checkpoints
A

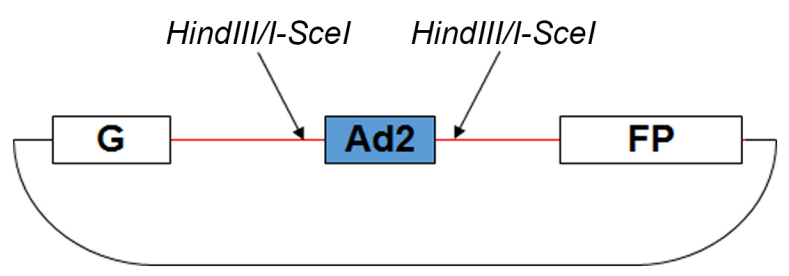

B

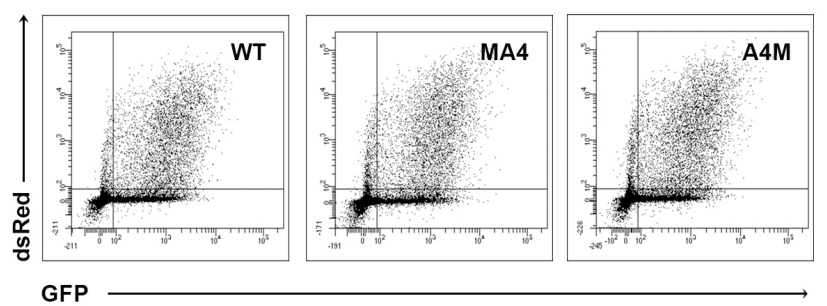

C

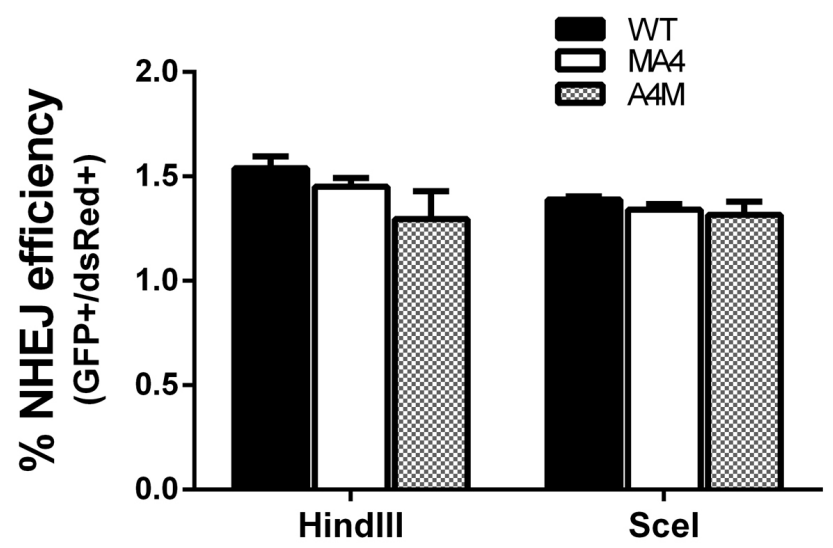

D

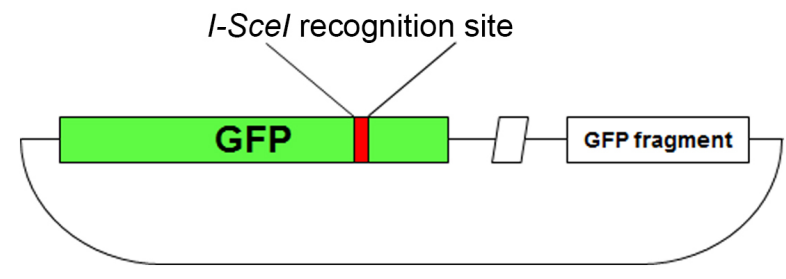

E

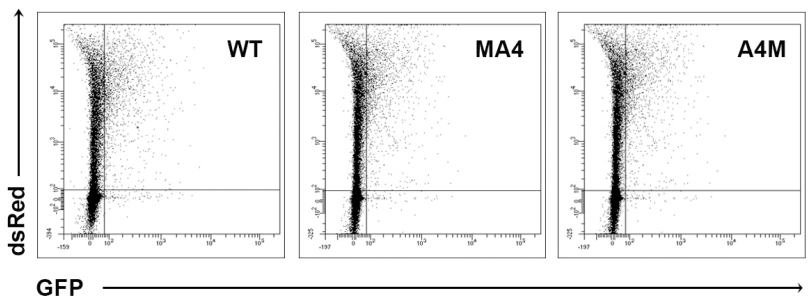

$\mathbf{F}$

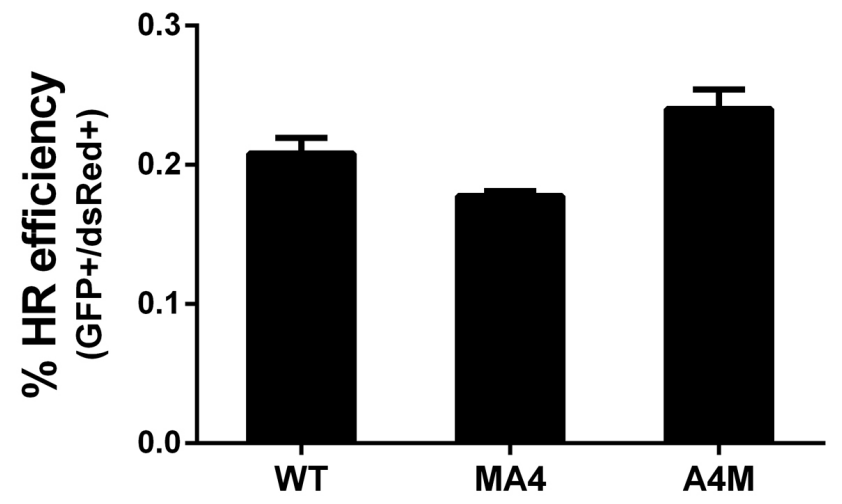

Figure 6: Analysis of NHEJ and HR in WT, MA4 and A4M-expressing cells. A. Schematic map of pEGFP-Pem1-Ad2 and pHR plasmids [38]. B. WT, MA4 and A4M cells were transfected with HindIII- or SceI-digested plasmid together with pDSRed2-N1 to normalize transfection efficiencies. GFP $v s$ DsRed flow cytometry data is shown $24 \mathrm{~h}$ after transfection. C. Percentage of NHEJ of HindIIIor SceI-digested plasmid for the three genotypes $(n=3)$. NHEJ efficiency was calculated as the ratio of GFP+/DsRed+ cells. D. Reporter plasmid used for detection of HR. E. WT, MA4 and A4M cells were transfected with SceI-digested HR plasmid together with pDSRed2-N1. GFP $v s$ DsRed flow cytometry data is shown $48 \mathrm{~h}$ after transfection. F. The ratio of GFP+/DsRed+ was used as a measure for HR repair efficiency. Data represents mean $\pm \mathrm{SD}$ of three independent experiments. 
which efficiently retain cells in G2 until they partially repair DSBs [22], and early G2/M checkpoint failure has been proposed as a molecular mechanism underlying etoposide-induced chromosomal aberrations [32]. A cell cycle arrest in $\mathrm{G} 2 / \mathrm{M}$ was achieved after etoposide treatment irrespective of MA4 and A4M expression, revealing a proper activation of the DNA damage checkpoints. A recent in vivo study has reported that rearrangements of the $M L L$ gene can only occur when cooperating defects in the DDR are in place. For instance, defective ATM, CHK2 and $\mathrm{p} 53$ signaling bypasses arrest of cells in G2/M phase, thus limiting the time for the cells to repair the damage before continuing to divide [11,32]. Because syngeneic cell lines were used it cannot be rule out that parallel or downstream insults cooperating with MLL fusions are also required to render further DNA damage vulnerability.

It has been very recently demonstrated that AML driven by repressive transcription factors, including AML1-ETO and PML-RAR $\alpha$ are sensitive to poly (ADPribose) polymerase (PARP) inhibition, due to suppressed expression of HR-associated genes and impaired DDR associated to prevention of binding of KU proteins to DNA ends in NHEJ $[33,34]$. In contrast, $M L L$-driven leukemias are proficient in DDR and insensitive to PARP inhibition. We found that MA4 and A4M do not regulate the expression of master proteins involved in the DNA repair pathways NHEJ, Alt-NHEJ and HR. Together, exposure to etoposide induces MLL fusions and preleukemic clone emergence but does not seem to facilitate the rapid acquisition of further mutations/DNA damage accelerating the clonal evolution to frank malignancy.

\section{MATERIALS AND METHODS}

\section{Plasmids construction}

pSpCas9(BB)-2A-GFP (PX458) plasmid was a gift from Prof. Feng Zhang (Addgene plasmid \#48138). Guide RNA against AAVS1 intron 1 was constructed using the primers AAVS1 Fw 5'-CACCGGGGCCACTAGGGACAGGAT-3' and AAVS1 Rv 5'-AAACATCCTGTCCCTAGTGGCCCC-3' [35]. pAAVS1.SA-2A-Puro-pA donor vector was obtained from Addgene (plasmid \#22075). pAAVS1.SA-2A-NeopA was made by replacing puro gene from pAAVS1.SA2A-Puro-pA using XhoI and $M f e I$ restriction sites. CAG promoter was PCR-amplified and cloned into both donor vectors using NotI/PacI restriction sites. A4M-GFP-SfiI and dTo-MA4-SfiI cassettes were subcloned in the Neo and Puro donor vector, respectively, by introducing in the donor vector a linker with two SfiI restriction sites (Figure 1A, 1F).

\section{Cell culture, transfection and antibiotic selection}

Human embryonic kidney 293 cells were maintained in Dulbecco's modified medium (DMEM; Gibco) supplemented with 10\% fetal bovine serum (FBS, Sigma), 1X GlutaMAX and $1 \%$ penicillin/streptomycin solution (Gibco), at $37^{\circ} \mathrm{C}$ and $5 \%$ of $\mathrm{CO}_{2}$. For gene editing experiments, $90 \%$ confluent 293 cells were transfected in 6-well dishes with $1 \mu \mathrm{g}$ pSpCas9 and $1 \mu \mathrm{g}$ donor vector using Fugene HD transfection reagent (Roche) following manufacturer's instructions. Two days after transfection, cells were selected with $1 \mu \mathrm{g} / \mathrm{ml}$ puromycin or $1 \mathrm{mg} / \mathrm{ml}$ G418 (Sigma).

\section{PCR detection of targeted homologous recombination}

In order to verify the CRISPR/Cas9-mediated integration in the AAVS locus, genomic PCR was performed using Taq DNA polymerase (Invitrogen) according to the manufacturer's instructions. To amplify the $5^{\prime}$ junction, two pairs of primers were designed inside the donor cassette (in the puro or neo gene) and outside the 5 ' homologous recombination (HR) region. Primers in the CAG promoter and outside the 3' HR region were used to amplify the 3 ' junction. Primers used are detailed in Table 1S.

\section{RNA extraction, cDNA synthesis, PCR and qPCR}

Total RNA was extracted using the RNAqueous kit (Ambion). Complementary DNA was synthesized using SuperScript III reverse transcriptase (Invitrogen). cDNA was used for conventional (MA4, A4M, GAPDH) and quantitative (MA4, HOXA9, PROM1) PCR. Conventional PCR was performed using Taq polymerase (Invitrogen) and quantitative PCR using Power SYBR ${ }^{\circledR}$ Green PCR (Life Technologies) and a CFX384 Touch $^{\mathrm{TM}}$ Real-Time PCR Detection System (BioRad). 2(- $\Delta \mathrm{CT}$ ) method was used to calculate the expression of the target mRNAs. Primers used are detailed in Table 1S.

\section{Southern blot}

Ten $\mu \mathrm{g}$ of genomic DNA extracted from HEK293 cells was digested with $B g l I I$ and separated on a $0.8 \%$ agarose gel by electrophoresis. The DNA was transferred onto a PVDF membrane and hybridized at $65^{\circ} \mathrm{C}$ overnight with a digoxigenin-dUTP-labeled probe. Primers used for probes are detailed in Table 1S. 


\section{Immunoblot}

$10^{6}$ cells were washed with PBS and resuspended in RIPA lysis buffer (Santa Cruz Biotechnology (SCBT)) containing protease inhibitors (Roche) or 2X Laemmli Sample buffer. Protein concentration was measured using the Bradford assay (BioRad) and samples were heated to $100^{\circ} \mathrm{C}$ for $5 \mathrm{~min}$. Protein samples $(20 \mu \mathrm{g} / \mathrm{lane})$ were subjected to SDS-PAGE and transferred to PVDF membrane (BioRad). After blocking in T-TBS buffer plus $5 \%$ bovine serum albumin, membranes were incubated with anti-human antibodies. The following primary antibodies were used: anti-phospho-Histone H2AX (1:1000) (Ser139 clone JBW301, Millipore), anti-KU70 (1:12000) (A9, mouse, Santa Cruz Biotechnology), antiKU86 (1:2000) (S10B1, mouse, Santa Cruz Biotechnology (SCBT)), anti DNA-PK ${ }_{\mathrm{cs}}(1: 1000)$ (rabbit, Abcam), antiXRCC4 (1:1000) (A7, mouse, SCBT), anti-DNA ligase III $\alpha(1: 1000)$ (1F1, mouse, Gene Tex), anti-WRN (1:1000) (H300, rabbit, SCBT), anti-PARP1 (1:1000) (rabbit, Calbiochem-Merck), anti-RAD51 (1:2000) (Rabbit, SCBT), and anti- $\beta$-Actin (1:100000) (mouse, SCBT). Horseradish peroxidase-linked donkey anti-rabbit or anti-mouse antibodies (SCBT) were used as secondary antibodies at 1:5000 dilution. Immunoblots were incubated for $1 \mathrm{~h}$ at RT and developed using ECL detection reagents (Amersham, Piscataway, NJ).

\section{MTT assay}

The effect of etoposide on cell proliferation was assessed by MTT assay $(n=3)$. Cells were seeded in triplicate in 96 -well plates at $2 \times 10^{5}$ cells/well. After $24 \mathrm{~h}$, etoposide (or vehicle) was added at increasing concentrations $(0-4 \mu \mathrm{M})$ for $3 \mathrm{~h}$. After a PBS wash, fresh medium was added and cells were incubated for 3 days. Then, $1 \mathrm{mg} / \mathrm{ml}$ MTT reagent (Sigma) was added and incubated for $3 \mathrm{~h}$. The absorbance was measured at 570 $\mathrm{nm}$ on an ELISA plate reader (Infinite 200, TECAN).

\section{Clonogenic survival assays}

For clonogenic survival assays, duplicates of 2000 cells for each condition were seeded in $100 \mathrm{~mm}$ dishes. After $24 \mathrm{~h}$, cells were treated with increasing concentrations of etoposide (or vehicle) for $3 \mathrm{~h}$. Cells were then PBS-washed, fed with fresh medium and maintained for 12 days. Cells were fixed and stained for CFU scoring with crystal violet solution $(0.5 \%$ crystal violet in $20 \%$ ethanol).

\section{Flow cytometry analysis of $\gamma \mathrm{H} 2 \mathrm{AX}$ and cell cycle distribution}

Half-million cells were seeded $24 \mathrm{~h}$ before treatment with $1 \mu \mathrm{M}$ etoposide (or vehicle) for $3 \mathrm{~h}$. Cells were then PBS-washed and harvested at the indicated time points following drug removal, fixed in cold $70 \%$ ethanol, and stored at $-20^{\circ} \mathrm{C}$ for $48 \mathrm{~h}$. Cells were then stained with a mouse anti-human anti-phospho $\gamma \mathrm{H} 2 \mathrm{AX}$ antibody (Millipore) followed by an anti-mouse-AlexaFluor-647 secondary antibody (Cell Signaling). Subsequently, cells were suspended in propidium iodide (PI) buffer containing $5 \mu \mathrm{g}$ PI and $100 \mu \mathrm{g} / \mathrm{mL}$ RNAase. Cell cycle distribution was analyzed on a FACSCanto-II cytometer using the FACSDiva software to discriminate among resting ( $\mathrm{G} 0 /$ G1) and cycling cells (S-phase and G2/M) [36].

\section{Non-homologous end-joining (NHEJ) and homologous recombination (HR) assays}

For these experiments HEK293T cells expressing either doxycycline-inducible MA4 or A4M were employed [37]. NHEJ and HR assays were performed as previously described [38]. Briefly, for NHEJ experiments $5 \times 10^{5}$ cells were doxycycline-induced $(1 \mu \mathrm{g} / \mu \mathrm{l})$ for 24 $\mathrm{h}$ and then transfected using Fugene HD transfection reagent (Roche) with $0.2 \mu \mathrm{g}$ of the normalizer plasmid pDSRed2-N1 and $0.5 \mu \mathrm{g}$ of linearized pEGFP-Pem1-Ad2 [39]. GFP and DsRed fluorescence were measured $24 \mathrm{~h}$ later using a FACSAria cytometer. A total of 50000 cells were analyzed. For HR assays, $2 \mu \mathrm{g}$ of the HR reporter construct were co-transfected together with $0.2 \mu \mathrm{g}$ of pDsRed-N1 using Fugene. GFP+ and DsRed + cells were quantified by flow cytometry $48 \mathrm{~h}$ after transfection. NHEJ and HR efficiency was calculated as the ratio of GFP+/ DsRed + cells [38].

\section{Comet assay}

Cells were irradiated with 40 Gy of ionizingradiation (IR) and were collected at different time points and processed for neutral comet assay as described previously [38]. Cell density was adjusted to $10^{5}$ cells $/ \mathrm{ml}$ in ice-cold PBS and mixed with LMAgarose (Trevigen, Gaithersburg, MD) at $37^{\circ} \mathrm{C}$ at a ratio of $1: 10(\mathrm{v} / \mathrm{v})$. Cell suspensions $(25 \mu \mathrm{l})$ were immediately transferred onto CometSlide slides (20-well slides) and placed at $4{ }^{\circ} \mathrm{C}$ for $10 \mathrm{~min}$. Slides were submerged in N1 lysis solution [38] and incubated overnight at $37^{\circ} \mathrm{C}$ in the dark. After rinsing in N2 buffer, slides were subjected to electrophoresis in $\mathrm{N} 2$ solution for $30 \mathrm{~min}$ at $1 \mathrm{~V} / \mathrm{cm}$. Cells were stained with ethidium bromide and analyzed with a fluorescence microscope (Zeiss Axioplan 2) equipped with a Hamamatsu Orca-EC camera. Images were obtained using 
Openlab software. At least 75 images per sample and experiment were analyzed. Tail moment was determined by the OpenComet software.

\section{Statistical analysis}

Data are expressed as mean \pm sd of independent experiments. Statistical comparisons were performed using either paired or unpaired Student $t$-test (GraphPad Prism software), as corresponding. Statistical significance was defined as a $p$-value $<0.05$.

\section{ACKNOWLEDGMENTS}

This work was supported by the European Research Council to P.M (ERC-2014-CoG-646903), MINECO (SAF2013-43065 to P.M), The Foundation Inocente Inocente and the Spanish Association of Cancer Research (AECC) to P.M and the Deutsche José Carreras Leukämie Stiftung to R.M/P.M. P.M also acknowledges the financial support from The Obra Social La CaixaFundaciò Josep Carreras and The Generalitat de Catalunya (SGR330). F.G. is supported by a Ramón y Cajal Grant (RYC-2014-16751) from the Ministry of Economy and Competitiveness (MINECO), Spain. N.C.G and A.B.H acknowledge financial support from The Cooperative Research Thematic Networks (RTICC) (RD12/0036/0058) and the INNOCAMPUS Program (CEI10-1-0010). We thank Dr. V Gorbunova (University of Rochester, New York, USA) for providing the pEGFP-Pem1-Ad2 and pHR plasmids.

\section{CONFLICTS OF INTEREST}

The authors have no conflict of interest to disclose. All authors have read and approved the manuscript in its present form.

\section{Editorial note}

This paper has been accepted based in part on peerreview conducted by another journal and the authors' response and revisions as well as expedited peer-review in Oncotarget.

\section{REFERENCES}

1. Meyer C, Hofmann J, Burmeister T, Groger D, Park TS, Emerenciano M, Pombo de Oliveira M, Renneville A, Villarese P, Macintyre E, Cave H, Clappier E, MassMalo K, Zuna J, Trka J, De Braekeleer E, et al. The MLL recombinome of acute leukemias in 2013. Leukemia. 2013; 27:2165-2176.

2. Bueno C, van Roon EH, Munoz-Lopez A, Sanjuan-
Pla A, Juan M, Navarro A, Stam RW and Menendez P. Immunophenotypic analysis and quantification of B-1 and B-2 B cells during human fetal hematopoietic development. Leukemia. 2015 Dec 29. doi: 10.1038/leu.2015.362.

3. Alexander FE, Patheal SL, Biondi A, Brandalise S, Cabrera ME, Chan LC, Chen Z, Cimino G, Cordoba JC, Gu LJ, Hussein H, Ishii E, Kamel AM, Labra S, Magalhaes IQ, Mizutani S, et al. Transplacental chemical exposure and risk of infant leukemia with MLL gene fusion. Cancer Res. 2001; 61:2542-2546.

4. Ross JA, Potter JD, Reaman GH, Pendergrass TW and Robison LL. Maternal exposure to potential inhibitors of DNA topoisomerase II and infant leukemia (United States): a report from the Children's Cancer Group. Cancer Causes Control. 1996; 7:581-590.

5. Wiemels JL and Greaves M. Structure and possible mechanisms of TEL-AML1 gene fusions in childhood acute lymphoblastic leukemia. Cancer Res. 1999; 59:4075-4082.

6. Felix CA. Secondary leukemias induced by topoisomerasetargeted drugs. Biochim Biophys Acta. 1998; 1400:233255 .

7. Hernandez A and Menendez P. Evidence linking pesticide exposure and pediatric leukemia: potential underlying mechanisms. International Journal of Molecular Sciences. 2016; 17. pii: E461.

8. Bueno C, Catalina P, Melen GJ, Montes R, Sanchez L, Ligero G, Garcia-Perez JL and Menendez P. Etoposide induces MLL rearrangements and other chromosomal abnormalities in human embryonic stem cells. Carcinogenesis. 2009; 30:1628-1637.

9. Libura M, Giebel S, Piatkowska-Jakubas B, Pawelczyk M, Florek I, Matiakowska K, Jazwiec B, Borg K, Solarska I, Zawada M, Czekalska S, Libura J, Jakobczyk M, Karabin K, Paluszewska M, Calbecka M, et al. Cladribine added to daunorubicin-cytarabine induction prolongs survival of FLT3-ITD+ normal karyotype AML patients. Blood. 2016; 127:360-362.

10. Moneypenny CG, Shao J, Song Y and Gallagher EP. MLL rearrangements are induced by low doses of etoposide in human fetal hematopoietic stem cells. Carcinogenesis. 2006; 27:874-881.

11. Nanya M, Sato M, Tanimoto K, Tozuka M, Mizutani S and Takagi M. Dysregulation of the DNA Damage Response and KMT2A Rearrangement in Fetal Liver Hematopoietic Cells. PLoS One. 2015; 10:e144540.

12. Gillert E, Leis $\mathrm{T}$, Repp $\mathrm{R}$, Reichel $\mathrm{M}$, Hosch $\mathrm{A}$, Breitenlohner I, Angermuller S, Borkhardt A, Harbott J, Lampert F, Griesinger F, Greil J, Fey GH and Marschalek R. A DNA damage repair mechanism is involved in the origin of chromosomal translocations $\mathrm{t}(4 ; 11)$ in primary leukemic cells. Oncogene. 1999; 18:4663-4671.

13. Reichel M, Gillert E, Nilson I, Siegler G, Greil J, Fey $\mathrm{GH}$ and Marschalek R. Fine structure of translocation breakpoints in leukemic blasts with chromosomal 
translocation $\mathrm{t}(4 ; 11)$ : the DNA damage-repair model of translocation. Oncogene. 1998; 17:3035-3044.

14. Strick R, Strissel PL, Borgers S, Smith SL and Rowley JD. Dietary bioflavonoids induce cleavage in the MLL gene and may contribute to infant leukemia. Proc Natl Acad Sci U S A. 2000; 97:4790-4795.

15. Eguchi M, Eguchi-Ishimae M, Knight D, Kearney L, Slany $\mathrm{R}$ and Greaves M. MLL chimeric protein activation renders cells vulnerable to chromosomal damage: an explanation for the very short latency of infant leukemia. Genes Chromosomes Cancer. 2006; 45:754-760.

16. Andersson AK, Ma J, Wang J, Chen X, Gedman AL, Dang J, Nakitandwe J, Holmfeldt L, Parker M, Easton J, Huether R, Kriwacki R, Rusch M, Wu G, Li Y, Mulder H, et al. The landscape of somatic mutations in infant MLL-rearranged acute lymphoblastic leukemias. Nat Genet. 2015; 47:330337.

17. Bardini M, Galbiati M, Lettieri A, Bungaro S, Gorletta TA, Biondi A and Cazzaniga G. Implementation of array based whole-genome high-resolution technologies confirms the absence of secondary copy-number alterations in MLLAF4-positive infant ALL patients. Leukemia. 2011; 25:175178.

18. Bardini M, Spinelli R, Bungaro S, Mangano E, Corral L, Cifola I, Fazio G, Giordan M, Basso G, De Rossi G, Biondi A, Battaglia C and Cazzaniga G. DNA copy-number abnormalities do not occur in infant ALL with $\mathrm{t}(4 ; 11)$ / MLL-AF4. Leukemia. 2010; 24:169-176.

19. Dobbins SE, Sherborne AL, Ma YP, Bardini M, Biondi A, Cazzaniga G, Lloyd A, Chubb D, Greaves MF and Houlston RS. The silent mutational landscape of infant MLL-AF4 pro-B acute lymphoblastic leukemia. Genes Chromosomes Cancer. 2013; 52:954-960.

20. Zhou BB and Elledge SJ. The DNA damage response: putting checkpoints in perspective. Nature. 2000; 408:433439.

21. Lobrich M, Shibata A, Beucher A, Fisher A, Ensminger M, Goodarzi AA, Barton O and Jeggo PA. gammaH2AX foci analysis for monitoring DNA double-strand break repair: strengths, limitations and optimization. Cell Cycle. 2010; 9:662-669.

22. Wei F, Xie Y, Tao L and Tang D. Both ERK1 and ERK2 kinases promote $\mathrm{G} 2 / \mathrm{M}$ arrest in etoposide-treated MCF7 cells by facilitating ATM activation. Cell Signal. 2010; 22:1783-1789.

23. Mladenov E and Iliakis G. Induction and repair of DNA double strand breaks: the increasing spectrum of nonhomologous end joining pathways. Mutat Res. 2011; 711:61-72.

24. Ceccaldi R, Rondinelli B and D'Andrea AD. Repair Pathway Choices and Consequences at the Double-Strand Break. Trends Cell Biol. 2016; 26:52-64.

25. Kowarz E, Merkens J, Karas M, Dingermann $\mathrm{T}$ and Marschalek R. Premature transcript termination, trans- splicing and DNA repair: a vicious path to cancer. Am J Blood Res. 2011; 1:1-12.

26. Caslini C, Serna A, Rossi V, Introna M and Biondi A. Modulation of cell cycle by graded expression of MLL-AF4 fusion oncoprotein. Leukemia. 2004; 18:1064-1071.

27. Gaussmann A, Wenger T, Eberle I, Bursen A, Bracharz S, Herr I, Dingermann T and Marschalek R. Combined effects of the two reciprocal $t(4 ; 11)$ fusion proteins MLL.AF4 and AF4.MLL confer resistance to apoptosis, cell cycling capacity and growth transformation. Oncogene. 2007; 26:3352-3363.

28. Wiederschain D, Kawai H, Shilatifard A and Yuan ZM. Multiple mixed lineage leukemia (MLL) fusion proteins suppress p53-mediated response to DNA damage. J Biol Chem. 2005; 280:24315-24321.

29. Breese EH, Buechele C, Dawson C, Cleary ML and Porteus MH. Use of Genome Engineering to Create Patient Specific MLL Translocations in Primary Human Hematopoietic Stem and Progenitor Cells. PLoS One. 2015; 10:e136644.

30. Buechele C, Breese EH, Schneidawind D, Lin CH, Jeong J, Duque-Afonso J, Wong SH, Smith KS, Negrin RS, Porteus $\mathrm{M}$ and Cleary ML. MLL leukemia induction by genome editing of human CD34+ hematopoietic cells. Blood. 2015; 126:1683-1694.

31. Torres R, Martin MC, Garcia A, Cigudosa JC, Ramirez $\mathrm{JC}$ and Rodriguez-Perales S. Engineering human tumourassociated chromosomal translocations with the RNAguided CRISPR-Cas9 system. Nat Commun. 2014; 5:3964.

32. Nakada S, Katsuki Y, Imoto I, Yokoyama T, Nagasawa M, Inazawa $\mathrm{J}$ and Mizutani S. Early G2/M checkpoint failure as a molecular mechanism underlying etoposide-induced chromosomal aberrations. J Clin Invest. 2006; 116:80-89.

33. Haince JF, Kozlov S, Dawson VL, Dawson TM, Hendzel MJ, Lavin MF and Poirier GG. Ataxia telangiectasia mutated (ATM) signaling network is modulated by a novel poly(ADP-ribose)-dependent pathway in the early response to DNA-damaging agents. J Biol Chem. 2007; 282:1644116453.

34. Paddock MN, Buelow BD, Takeda S and Scharenberg AM. The BRCT domain of PARP-1 is required for immunoglobulin gene conversion. PLoS Biol. 2010; 8:e1000428.

35. Mali P, Yang L, Esvelt KM, Aach J, Guell M, DiCarlo JE, Norville JE and Church GM. RNA-guided human genome engineering via Cas9. Science. 2013; 339:823-826.

36. Munoz-Lopez A, van Roon EH, Romero-Moya D, LopezMillan B, Stam R, Colomer D, Nakanishi M, Bueno C and Menendez P. Cellular Ontogeny and Hierarchy Influence the Reprogramming Efficiency of Human B-Cells into Induced Pluripotent Stem Cells. Stem Cells. 2016.

37. Benedikt A, Baltruschat S, Scholz B, Bursen A, Arrey TN, Meyer B, Varagnolo L, Muller AM, Karas M, Dingermann $\mathrm{T}$ and Marschalek R. The leukemogenic AF4-MLL fusion protein causes $\mathrm{P}-\mathrm{TEFb}$ kinase activation and altered 
epigenetic signatures. Leukemia. 2011; 25:135-144.

38. Herrero AB, San Miguel J and Gutierrez NC. Deregulation of DNA double-strand break repair in multiple myeloma: implications for genome stability. PLoS One. 2015; 10:e121581.
39. Seluanov A, Mittelman D, Pereira-Smith OM, Wilson JH and Gorbunova V. DNA end joining becomes less efficient and more error-prone during cellular senescence. Proc Natl Acad Sci U S A. 2004; 101:7624-7629. 